\title{
A Peircean approach to pictorial documents
}

\author{
Tony Jappy, \\ VECT, University of Perpignan Via Domitia, \\ France
}

[Logic] is in short The Philosophy of Representation (CP 1.539)

\begin{abstract}
The paper summarizes six chapters of a book introducing Peircean visual semiotics to non-specialists. The book has an epistemological bent, and is intended as an empiricist response to Saussurean rationalism, locked away as it is in the universe of Thirdness. Inevitably, in reducing two hundred and thirty pages to twenty-four, the paper has been shorn of the majority of the original examples, quotations and summaries, the relations between the various chapters (here sections) are not developed in detail and the general presentation is perforce allusive: for example, the dynamic object, surely one of Peirce's most potent yet least understood concepts, has been simplified in what is, after all, an introductory text; similarly, acquaintance with his system of categories has largely been taken for granted. Finally, Simonides of Ceos' epigram stating that a poem is a talking painting and a painting a mute poem gives the book its title and a major leitmotiv, namely the way we obtain information from pictorial documents.
\end{abstract}

Most semioticians are by now familiar with the fact that Peirce considered his semiotics as a species of logic, and the idea that the interpretation of signs, beautiful or otherwise, should fall to a discipline as abstract as logic has caused concern and dismay among theorists of the sign interested in language, literature and pictorial artifacts. However, as a proto-cognitive scientist concerned with the nature and acquisition of scientifically valid knowledge, Peirce not only set his research within mathematics and phenomenology, but was also attentive to the way the rules and theorems of science were to be represented. He thus dignified this aspect of scientific inquiry with the status of a philosophy: his semiotics, therefore, as we know it, turns out to be but the first of three separate branches of logic, branches which he considered collectively as the "philosophy of representation".

What follows, then, is an attempt to give the flavor of this particular conception of philosophy as it applies to pictorial documents. It is a summary of a recent book which deals specifically with this form of logic: a visual semiotics, in other words (Jappy 2010). Isolating this particular aspect of the general theory will possibly irk the specialist, but as the book in question was published in French, it seemed that non-francophone semioticians might be interested in an English version of the major points of the research. 
The article deals in turn with the three divisions of the 1903 classification of signs, plus what can be considered one of the core elements of his philosophy of representation, namely the relation between the sign and the medium through which it is communicated; but first a section on certain important non-mimetic considerations and, quite obviously, the definition of the Peircean sign. References to Peirce's definitions, etc., employ the customary presentation of paragraphs from the Collected Papers (e.g. CP 2.277) or to page numbers in volume two of the Essential Peirce (e.g. EP2 477).

\section{Non-mimetic considerations}

This section introduces some of the important general "pre-semiotic" properties of images to which reference will subsequently be made. They have theoretical as well as methodological implications, and are prerequisites for a semiotics of pictorial data. We begin with some very basic differences between image and text, namely their respective "dimensionalities", a theoretical construct first introduced in the $18^{\text {th }}$ century; these are followed by the communication channels that pictorial media exploit, and, finally, by an important distinction between frame and window.

\section{Bodies and actions}

One of the first modern, semiotically-oriented, discussions of the differences in dimension that distinguish text and image is to be found in the $18^{\text {th }}$ century study Laocoön (Lessing 1766 [1984]). Although the thematic basis of the theory of aesthetics that Lessing develops in this work is a three-dimensional statue, his principal concern is with the way the two dimensions of painting and the single dimension of poetry motivate by a "suitable relation" each art form's representational potential. Drawing on Aristotle's theory of imitative creation, he formulates these distinctions "from first principles" in a well-known passage at the beginning of chapter sixteen. Substituting "text" for "poetry" and "image" for "painting", the upshot, in Lessing's view, is that the one-dimensional line of language units composing texts makes for inherently dynamic, progressive representations of actions, while the two-dimensional still image is a space on which bodies extend in a static "flat". These distinctions can conveniently be illustrated by Plate 1, a frame from a mid-twentieth century British comic strip, combining text and pictorial "bodies" in the cropped representations of two adolescents, a pair of spectacles and a wig.

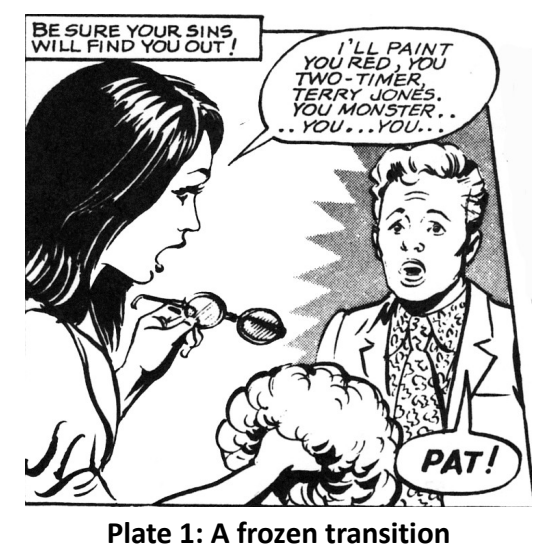

Several paragraphs later Lessing also suggests that bodies, not only in the most general sense, but also in the particular case of human bodies, change over time as a result of actions in series. However, he notes, the state of a body, e.g. a human face and its expression, is, at any 
given moment, the result of some prior action, and no doubt will be the cause of some subsequent change of face and facial expression.

\section{Channels of communication}

In its complete form the comic-strip is multimodal. Nevertheless, its dominant mode of representation is pictorial, or, more technically, iconic, while the information we derive from the vignette as we interpret it is conveyed, not by two, but by at least three distinct communication channels. The first channel is the pictorial, nonverbal channel, the nature of the information which it provides being the subject of a later discussion. This channel, composed basically of lines, forms and, generally, colors, occupies the surface of the image, and this particular example illustrates the way any still image can be interpreted as a sort of frozen transition between prior and subsequent events in the strip.

The second channel is, of course, the verbal. This is the information channel with which we are most familiar, not only in its written form but also through conversation and oral discourse generally, and it is, of course, the channel without which we could barely survive. This verbal channel obviously has an ancillary role to play in the understanding of comic strips and films, etc., in that such sequences of images contain dialogue, but in no way does the verbal channel constitute the dominant mode that it would assume in a poem or a work of fiction, for example.

A third, circumstantial, source of information is supplied by the communication channel conveying well known mass media conventions, such as the placing of authorial intrusion in oblong boxes in the top left corner of selected frames, a position from which the western eye has been accustomed to scan any two-dimensional document. Were this a traditional Japanese manga or a comic-strip drawn by an Arab graphic artist, this top-left to bottom-right orientation would, like the verbal material it contains, be oriented in the opposite direction. There are, in addition, pictorial conventions - varieties of "balloons" - to indicate speech, thoughts, telephone conversations, etc., a jagged halo here to suggest surprise, a general leftto-right orientation of characters indicating which character initiates dialogue and action.

\section{Frame and window}

Now if the two-dimensional structure of the image makes available a flat surface on which to inscribe various types of "bodies", as Lessing has it, it also offers the possibility of closure by means of a frame - not simply a frame in the traditional sense, but a frame as described by Meyer Schapiro in a remarkable discussion of field and vehicle in pictorial signs in one of the founding texts of a general visual semiotics. The frame around a painting, he suggests, is "like a window frame through which is seen a space behind the glass. The frame belongs, then, to the space of the observer rather than the illusory, three-dimensional world disclosed within and behind", (Schapiro 1994: 7). While comic-strips are not at all at issue in his essay, Schapiro is nevertheless drawing attention to the fact that when we interpret a picture, or a vignette such as Plate 1, we, the observers, unconsciously enter the three-dimensional world of the protagonists, no less than when we become involved in, and are moved by, a telefilm on TV or a film at the cinema. Over and above its theoretical implications, Schapiro's farreaching remark suggests a methodology for a semiotics of pictorial representations.

Firstly, it should never be forgotten that the "space behind the glass", e.g. the threedimensional world in which the protagonists of the frame are enacting their teenage drama, is 
a world apart, and should be kept so in any semiotic study of images. This is the mute world of the purely pictorial with its specific range of signs. Secondly, we must take into account that the frame itself, as Schapiro suggests, belongs to the space of the observer, but above all to the space of the graphic artist who, for various reasons, produced the comic-strip in the first place. His is the world or space of the third communication channel, and his lines of conduit, so to speak, are delimited by the frame. This means, of course, that the succession of events which includes the one depicted on Plate 1 is determined by two agencies: the graphic artist, whose moral purpose we can divine from the plot ("Be sure your sins will find you out") and the more local determinations of the characters as they go about their adolescent intercourse "behind the glass". We note, at this point, that the verbal material in the strip belongs on both sides of the frame: it grounds the dialogue of the protagonists and is the vehicle for the authorartist's contextual cues in the third communication channel.

It follows, then, from a non-mimetic point of view, that a visual semiotics concerns itself not only with the first and last of the communication channels mentioned above, but also, separately, with the frame and the "contents" of the window: in short, with, what is left in a (still) image once all the verbal material has been excised.

\section{Sign and sign action}

This section briefly introduces the basic constituents of the theory of sign action, or semiosis, a logical relation holding between three correlates developed by Peirce at the beginning of the $20^{\text {th }}$ century (CP 2.233). These correlates are the sign itself, the "cause" of the sign, named the dynamic object, and, finally, the effect that the sign produces on the person interpreting it, namely the interpretant.

\section{Semiosis}

This is how Peirce defined the sign and its relation to the other two correlates in 1908:

I define a Sign as anything which is so determined by something else, called its Object, and so determines an effect upon a person, which effect I call its Interpretant, that the latter is thereby mediately determined by the former. My insertion of "upon a person" is a sop to Cerberus, because I despair of making my own broader conception understood (CP 8.343)

The influence on his conception of semiosis of the three categories can clearly be seen in the following militaristic example of semiosis that Peirce himself offers:

Suppose, for example, an officer of a squad or company of infantry gives the word of command, "Ground arms!" This order is, of course, a sign. That thing which causes a sign as such is called the object (according to the usage of speech, the "real", but more accurately, the existent object) represented by the sign: the sign is determined to some species of correspondence with that object. In the present case the object the command represents is the will of the officer that the butts of the muskets be brought down to the ground. (CP 5.473)

We note the three-term structure of the order given to the squad of soldiers and, just as importantly, the fact that while the order itself belongs to the world of actuality and existence (Secondness), the object belongs to the altogether more general realm of volition and thought (Thirdness). Note, too, that the example also makes the important point that in the course of 
semiosis, the sign is in some way "determined to some species of correspondence" with its object. In other words, the nature of the object determines the structure of the sign (structure being a case of Firstness); alternatively, we could say that whatever structure a sign displays, it inherits it in some way from its object. A simple linguistic example shows how this might be the case: the example of semiosis above contains an order, and this determines the distinctly injunctive structure of the sign. If, on the other hand, the colonel had wanted to communicate information to the orderly, the syntax of the sentence would have had the completely different form of a declarative.

Returning to the pictorial example on Plate 1, it should be obvious that the effect, or interpretant, of the sign formed by the expression on the girl's face, her verbal outburst and the removal of the wig and glasses, is the character called Terry's reaction: a reaction which our experience of the world has taught us to recognize, and which is here communicated via the three communication channels identified above, namely the recognizable expression of surprise on his face, the exclamation of the proper noun "Pat!", and the cartoon artist's "surprise" signal effected by the jagged halo round his head and shoulders suggesting a recoiling movement. Since he registers surprise at Pat's removing the wig and glasses, it follows that this plus her angry outburst correspond to the complex sign which triggers his reaction. What caused her to remove her wig and spectacles is the object of this particular sign, and obviously originates in the previous vignette. These, then, constitute, in the protagonists' world at least, the object of the sign visible in the image.

The functioning of semiosis can be illustrated schematically by means of Figure 1, where the arrows indicate the direction of determination - the semiotic "determination flow", so to speak - from the object to the interpretant via the sign. The broken line between the object and the interpretant indicates the impermissible immediate relation between object and interpretant (i.e. a relation not mediated by the sign), the sort of relation which might hold in some outlandish theory of telepathy. On the other hand, the solid horizontal line separating the sign from both object and interpretant illustrates the fact that the sign, e.g. the order given to the soldiers, belongs to the existential world of the troughs and crests of the air waves of speech, for example, while the object and interpretant belong to the phenomenologically more complex and general world of thought and desire. This latter feature of sign action, namely, differences of complexity concerning the object and the interpretant on the one hand and the sign itself on the other - a consequence of Peirce's three-way categorical distinctions - is more properly seen as a function of the ecology of signs, and will be dealt with in greater detail below.

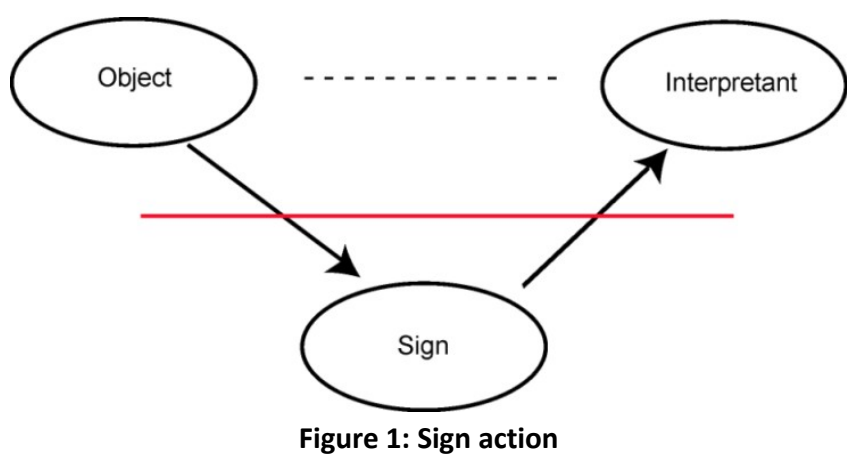




\section{Two objects}

Obviously, at one level the particular arrangement of lines and shapes on Plate 1 composes a sign representing a lovers' tiff: we recognize the purely graphic elements of the sign as representing as its objects expressions and postures of anger and surprise. In other words, anger and surprise are the determinants of this particular configuration of lines and forms, etc. However, at another level, if these particular facial features are there at all it is necessarily as a consequence of the moral purpose of the author-artist who chose to put them there in the first place (more properly stated, they are the consequence of the relationship between this particular ontology or universe of existence playing out behind the glass and the artist's more or less momentary state of mind: see CP 8.178). It follows, then, that the process of "determination by an object" in images (and in verbal communication, too) is extremely complex and must exist on at least two levels. At one level, behind the glass, as Schapiro puts it, we enter a denoted world of causes and effects represented by the visible objects on the page; on another level, on our side of the frame, we are part of the space of the author-artist who drew them there in the first place. And thus it is that in the comic-strip, as in any other pictorial sign - in any sign, in fact - there seem to be two distinct types of objects engaged in this complex semiotic activity, those constituting the participants in the represented adolescent world of mistrust and deceit, the other belonging to the space of the author-artist and to ours, the readers.

This potentially misleading situation was resolved in 1904 when Peirce reasoned that semiosis involved in fact not one but two distinct types of object: firstly, the less visible object responsible not only for the sign on Plate 1 but for all the others in the strip, too, namely, the intentions of the artist; and secondly, the object that we infer and identify by experience as the face of an angry young woman confronting a surprised young man (each represented by a specific configuration of lines). The first type of object he termed the "dynamic" object, which is the object outside the sign; that is, the object which instigates a sign in the first place and determines it to be what and such as it is. The second he called the "immediate" object, the object within the sign, the "trace", so to speak, of the first object as it occurs in this particular vignette, namely the two adolescents, the spectacles and the wig, etc.

We note, finally, that these two objects correspond conveniently with Schapiro's distinction between frame and window: the dynamic object belongs to the space of the frame, while the immediate object is formed from the visible world of the protagonists behind the glass. Thus, while the immediate objects of any (figurative) painting or photograph, for example, are what we see in the image, the dynamic object has to be inferred collaterally from the hints left more or less consciously by the artist, photographer, cinematographer, etc.

\section{How shall the sign be called?}

The first of Peirce's 1903 divisions identifies the three major subclasses of signs we are likely to encounter in our everyday affairs. In an illuminating passage in "Pragmatism", a text from 1907, he writes of the subclasses in question as being, respectively, "of the nature of a significant quality, or something that once uttered is gone forever, or an enduring pattern like our sole definite article" (EP2 403). Although less well known, this division, which he had earlier divided into qualisign, sinsign and legisign, is no less pertinent to the analysis of images than the better known second division distinguishing between icons, indices and 
symbols: furthermore, the signs we find in images of all types, and indeed the images themselves, correspond no less to this three-way distinction than the more familiar verbal examples Peirce gives.

As Schapiro's perspicuous observation urges us to, in dealing with pictorial documents, we must be careful to distinguish between the sign itself and its "contents", and nowhere is this more evident than in the first division. To see just how this applies, consider how the following photographic representation of jubilant soccer fans compares with the teenagers from Plate 1 above:

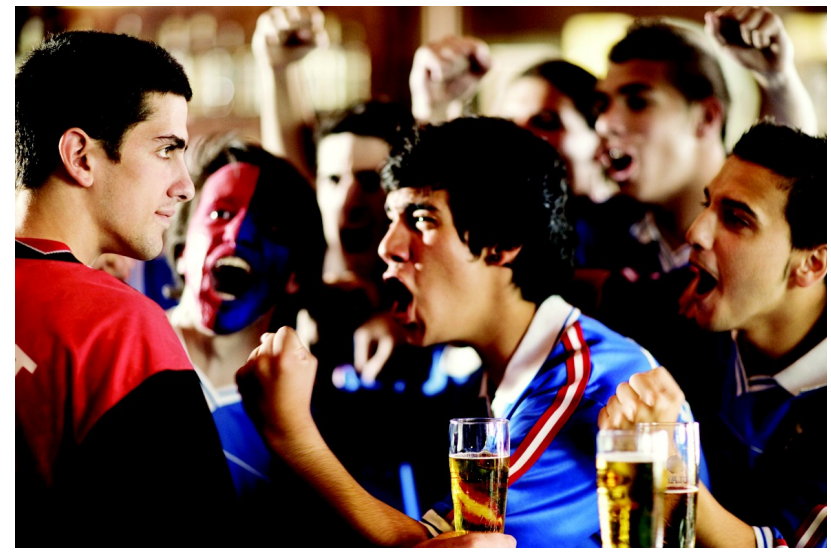

Plate 2: Rival soccer fans

Clearly, the photograph of the soccer fans and the sketch of the teenage lovers in Plate 1 are in themselves "singular" signs, in the sense that they are singular events, that is, one-off signs, the material making of which, like Peirce's once-uttered sign, is over and gone forever. They are infinitely reproducible but can never be repeated in exactly the same way anywhere else or at any other time, and are in no way part of a system or "enduring pattern". This is because as "frames" they are the individual creations of the existential spaces of the photographer and the graphic artist: photographs and sketches are thus sinsigns. On the other hand, signs like those exhibited within the space of the protagonists in either illustration, both the verbal and the nonverbal - gesture, expression, etc., specificity of appearance - are general and systematic to the extent that they can be used with the same "meaning" in diverse combinations in an infinite variety of situations, and are clearly more complex in that their interpretation is governed by general rules which we learn from childhood, and as such transcend individuality. They are, therefore, signs which in a certain manner are law-governed: mass media conventions - cropping, framing techniques, etc.- and the various systems of nonverbal communication signs exhibited by these examples are all, no less than verbal signs, types of legisign, and, although less complex and less easily articulated, require careful identification.

\section{Nonverbal communication legisigns}

For convenience, in describing such nonverbal legisign systems it is useful to adopt the classifications proposed by certain social psychologists, whose research bears upon the ways in which human verbal communication is embodied in, and abetted by, precisely these various nonverbal sign systems in real life, of which we have two pictorial representations here. The exact typology of nonverbal communication (NVC) systems varies from author to author. Noted early research in the field was conducted by the American anthropologist, Edward Hall, 
whose work on "proxemics", i.e. the study of the signification of distances maintained between humans as they interact, was taken up and systematized as a form of "body language" by the French lexicologist and semiologist, Pierre Guiraud (e.g. 1980). An excellent but relatively dated selection of articles on the semiotic aspects of real life (i.e. not necessarily represented pictorially) "somatic" nonverbal communication is to be found in Kendon (1980). Of special interest is Kendon's introductory essay. However, for the sake of simplicity and consistency, I adopt here the typology of the social psychologist, Michael Argyle (1972, 1988).

In his work, Argyle postulates a certain number of (generally culturally variable) nonverbal signals, which are, in effect, behavioral legisign systems, and several of these figure prominently in the photograph and vignette examined above. Some of the more visually salient of these are often scalar signals, precisely the sort likely to be reproduced as signs in pictorial media. Remember, once more, that social psychologists, ethnologists and anthropologists, like many semioticians, study such legisign systems in real life human interactions, whereas visual semiotics tends to examine and classify such signs as they are represented pictorially at one remove from reality in confrontations, for example, behind the semiotic "window". On Plate 2 above we find, among other NVC legisign signals: proximity (Hall's proxemics), facial expression, "war paint" on the face, gesture, posture, orientation, and perhaps most notably at a soccer match, appearance, which is a potent way for the protagonists to send out information about themselves. To these must be added, of course, the numerous mass media conventions encountered in image-making of all kinds.

We note, at this point, that there remains one subclass of sign still to be described, one that is such that it cannot be illustrated in isolation qua sign, and is the least complex within the current division. Such signs are the qualitative components to be found in the material sinsigns "higher up the scale", so to speak, and as such are, in themselves, pure potentialities. These are what enable us to identify the particular expressions of the two teenage lovers, and the attitudes of the soccer fans and their lone opponent: they generally occur in clusters of qualities, thereby enabling us to interpret them as surprise and guilt in one or jubilant, menacing behavior in the other. Simplifying considerably, we might say that these qualities or properties constitute the lines, shapes and colors of which all pictorial signs are composed, and we only perceive them to the extent that they inhere in such signs and make it possible to discriminate between them. They are simply abstract qualities, qualities in limitless diversity which function as signs, the very stuff, for example, of wine tasting, perfume confection and piano-tuning and, as here, image-making. Such are the qualisigns.

Finally, there are two important aspects of the classification that must be borne in mind. Firstly, implicit in this first division, but explicitly stated elsewhere (see below), is the fact that the subclass lower down the scale is involved in the subclass above. All sinsigns have a semiotic identity guaranteed by their specific set of qualisigns. Secondly, the legisigns themselves are general and immaterial, and so cannot be perceived as such, whence the horizontal line separating the sign from its object and interpretant on Figure 1 above: they are materialized and appear to us through the existent nature of the medium as a special class of sinsign which Peirce named replicas. Legisigns, both verbal and, as in Plates 1 and 3, nonverbal, govern innumerable instances; these, the replicas, are characterized by their specific clusters of qualisigns enabling us to identify them when we encounter them both in 
texts and images and in the infinite variety of encounters we make in real life. Just how the representation of these replicas differs in a line drawing and a photograph (Plates 1 and 3, respectively) is a topic to which we now turn.

\section{Modes of representation}

"A sign", wrote Peirce in 1911, "is either an icon, an index or a symbol" (CP 2.304), and we attend now to this particular facet of signs, namely, the three modes of representation which constitute the second criterion for their classification. It concerns the three principal ways in which a sign can represent its object; or, what amounts to the same thing from a different point of view, it concerns the nature of the relation holding between the sign and the object which causes it to exist in the first place. First established by Peirce in 1867, the three-way distinction between icon, index and symbol is by far the best known and most widely canvassed, even figuring paradoxically in the theoretical arsenal of competing semiotic theories and in most accounts of visual culture; within it, the concept of the icon, based as it is on the relation of resemblance, is probably the single most controversial subclass established by Peirce. This division constitutes the second in the triadic system of 1903, following the distinction between qualisigns, sinsigns and legisigns examined above, and preceding the "informational" division to be examined in the final section below.

From the point of view of a visual semiotics, the icon is probably the most interesting mode of representation, for as the name suggests, iconic signs are composed of "significant qualities", Firstnesses therefore, and it is not immediately evident how they can acquire the Thirdness status of symbols. Initially, Peirce called such signs "likenesses", but later adopted the universally known, but much contested, "icon". In a way, this is unfortunate as the term, from a Greek word for "image", suggests that this subclass deals simply with types of picture, whereas the qualitative nature of the icon is valid not only for vision but also for smell, touch, taste and hearing: for example, the notes of music we hear in the concert hall are no less iconic, in the strictly Peircean sense, than a painting of Marlborough Castle-they simply involve a different set of significant qualities.

In what follows the three subclasses are described in order of increasing complexity and, to give the reader the flavor of Peirce's original formulations, the most important definitions are introduced as the argument develops. Given the controversial nature of this particular division, and in view of the importance the concept of the icon has in a discussion of visual semiotics, we begin with a number of general considerations which should lead to a better understanding of why Peirce associated the relations of resemblance, physical contact and convention with, respectively, icon, index and symbol.

Herewith another highly important definition of the sign, dating from 1906:

I use the word "Sign" in the widest sense for any medium for the communication or extension of a Form (or feature). Being medium, it is determined by something, called its Object, and determines something, called its Interpretant [....] In order that a Form may be extended or communicated, it is necessary that it should have been really embodied in a Subject independently of the communication; and it is necessary that there should be another subject in which the same form is embodied only as a consequence of the communication. (EP2 477) 
In this passage from a letter to Lady Victoria Welby, Peirce is expounding on an important aspect of the nature of the relations holding between the sign and object, and in particular, on the formal consequences of the determination of the sign by the object, a principle already encountered in Peirce's militaristic illustration of semiosis discussed above. The interesting point, for it constitutes one of the major theorems of linguistic iconicity theory, is that in determining the sign to existence, the object imparts or communicates part of its form to that sign: in an older sense of the term, we might say that the object informs the sign, whence the concept of "information". For obvious reasons this principle is particularly evident in the case of the least complex of the present group, the icon.

\section{The icon}

Plate 1, the vignette examined above minus the verbal elements, is offered as a typical example of the icon. The relation between the sign and what it represents is necessarily qualitative, since the former is composed simply of black lines and shapes: for us to be able to identify as teenagers the persons represented, both object and sign must have at least these qualities in common; and the nature of the relation between the two is one of resemblance, since, for Peirce, any two entities can be said to resemble each other if they share at least one quality. Such a sign can offer no proof of the existence of the protagonists, its immediate objects: as the reader can see for himself, they simply look like teenagers. The fact that we recognize them as such is simply a matter of experience, and has nothing to do with the sign itself: even a child would recognize it as representing two quarrelling youngsters for the simple reason that this is what they look like. From our experience, "the cognitive resultant of our past lives", we construct inferentially a composite image from the lines and shapes on the page.

Plate 1, then, is an example of an icon, a sign which is said to be "motivated", that is, determined in its very shape by part of the structure of the object that it represents in the way described in the definition given above (EP2 477). The following is Peirce's painstakinglyformulated definition:

An Icon is a sign which refers to the Object that it denotes merely by virtue of characters of its own, and which it possesses, just the same, whether any such Object actually exists or not. It is true that unless there really is such an Object, the Icon does not act as a sign; but this has nothing to do with its character as a sign. Anything whatever, be it quality, existent individual, or law, is an Icon of anything, in so far as it is like that thing and used as a sign of it. (CP 2.247)

By definition, then, an icon is a sign that signifies by virtue of the fact that it shares at least one "character" or quality with the object that determines it (though such a simple sign, it must be said, would have very little practical interest, should it exist). In other words, when the sign and its object simply share common qualities or properties such as lines and shapes, etc., as in the sketch above, then the sign is an icon of that object, and its characteristic representative quality is to be such as it is, independently of both object and interpretant. To the extent, then, that an entity has at least one quality it is fit to function as a sign, though it cannot do so until it conforms to some object and has determined in turn an interpretant. 


\section{The index}

Here is how Peirce defined the index in 1903, in a passage following the definition of the icon given above:

An Index is a sign which refers to the Object that it denotes by virtue of being really affected by that Object. It cannot, therefore, be a Qualisign, because qualities are whatever they are independently of anything else. In so far as the Index is affected by the Object, it necessarily has some Quality in common with the Object, and it is in respect to these that it refers to the Object. It does, therefore, involve a sort of Icon, although an Icon of a peculiar kind; and it is not the mere resemblance of its Object, even in these respects which makes it a sign, but it is the actual modification of it by the Object. (CP 2.248; emphasis added)

The semiotic structure, or constitution, of the index thus necessarily involves two elementssign and object: in other words, this constitution is dyadic. In addition to the definition it offers of the index, the passage makes a further important point: by virtue of the principle of implication underwritten by the relations holding between his three categories, Peirce is able to justify theoretically just why it is that an index such as a photograph is interpretable at all. The physical contact between object and film is, in itself, only temporarily observable (if at all, though in the case of film the actors and technicians, etc., all witness the indexical nature of the filming process). Nevertheless, the "impact" of the object on the film is such that it leaves a complex but visible mark or trace which contains information of a sort concerning the "model" that brought it into being. In other words, just as any Secondness requires qualities of Firstness to give it its specific identity, so too the index has an icon "nested" within it, an icon without which we should not be able to identify the perceptible immediate object of the sign or the external object it represents.

Whereas in the case of the icon the existence of the object was not a prerequisite for its own particular representative quality - it was simply required to possess at least one quality - the index is defined precisely by the fact that the object is necessarily involved in the dyadic structure of its semiotic constitution: the interpretant, therefore, does not enter into the picture, though indices obviously do not function as such until they actually determine an interpretant. Consider, as an example, Plate 2 above, the photograph of the soccer fans. Since, in Peirce's words, the sign is really (i.e. existentially, physically) "affected by its object", he frequently proposed the photograph as an excellent illustration of the index, if only for the simple reason that it is not possible, or wasn't in the days of argentic photography, to take a photograph of a non-existent object, a condition which clearly shows the relation between sign and object to be existential. This means that an index can only relate to an individual object, or, as in the example or in the case of a group portrait as in a school photo, to a group of individuals, to a group of "particulars". Such a sign is deictic, i.e. has a pointing, designating function inasmuch as it relates to, and is only understandable in relation to, a unique individual (or group of individuals), to a unique time and to a unique place: 'The index asserts nothing; it only says "There!" It takes hold of our eyes, as it were, and forcibly directs them to a particular object, and there it stops' (CP 3.361).

Peirce furthermore makes the very important point that since the actual physical contact (a contact which we don't normally witness) between sign and object is what makes the sign indexical, in order for us to recognize the person whose photograph it is, the index must 
incorporate an icon. Putting this implication principle back to front, he states: "A photograph, for example, not only excites an image, has an appearance, but, owing to its optical connection with the object, is evidence that that appearance corresponds to a reality" (CP 4.447). In the present case, this "image" or "appearance", namely the iconic material in the photograph, is a very much more detailed composition of lines, shapes and colors than the sketch on Plate 1, but is nothing more, nevertheless, than the iconic set of traces of the shapes and colors reflected by the jubilant fans and their hapless rival.

In similar fashion, a footprint in the sand, an index, will indicate that some animal or other has walked in the area, but the actual shape of the footprint, its iconic content, will enable us to determine whether it was made by a crab, a bird or, as Robinson Crusoe discovered, a human being. It thus follows that an index, too, is a motivated sign.

Finally we note that while the vignette on Plate 1 as a whole is an iconic sinsign, it nevertheless represents, behind the glass, iconic replicas of the relatively simple NVC legisigns described above: expression, orientation, proximity, appearance, etc. The photograph on Plate 2 also contains the representation of similar replicas of NVC legisigns. These, too, are iconic - they constitute the visible material of the sign, and it is not because the photograph is indexical that the status of what we see in it should also be indexical: to be perceived and interpreted at all they require the qualitative contribution of the icon. On the other hand, what we see as iconic material would, in the actual confrontation, be experienced by the protagonists as very real indices of two warring soccer tribes. This is the case too in cinematography: (with the exception of animation), the material production of the film is indexical but what we see on the screen is purely iconic.

\section{The symbol}

Finally, the most complex relation of all holding between a sign and its object is one that transcends individuality and represents a very general object. In this case, since no individuals are involved, only classes, the sign has to be learned (its object can't be seen in the way that an individual can, and has to be inferred), and functions therefore by convention, by "agreement" among the sign users. This means that such signs call for the active participation of the user/interpreter - anyone who doesn't already know what the word legisign means is not likely to understand it when they first come across it, unless the meaning can be inferred from the context. Common nouns, verbs, adjective and adverbs are all linguistic examples of symbols adduced by Peirce, while proper nouns, demonstratives, personal and relative pronouns, etc., are all examples of a sub-category of indexical legisigns he termed hyposemes. However, as mentioned above, it is an interesting question within a purely visual semiotics how predominantly qualitative nonverbal signs like icons might acquire the complex status of symbolhood.

To begin with, this is how Peirce defines the symbol:

A Symbol is a sign which refers to the Object that it denotes by virtue of a law, usually an association of general ideas, which operates to cause the Symbol to be interpreted as referring to that Object. It is thus itself a general type or law, that is, is a Legisign. As such it acts through a Replica. Not only is it general itself, but the Object to which it refers is of a general nature. Now, that which is general has its being in the instances which it will determine. There must, therefore, be existent instances of what the 
Symbol denotes, although we must here understand by "existent," existent in the possibly imaginary universe to which the Symbol refers. The Symbol will indirectly, through the association or other law, be affected by those instances; and thus the Symbol will involve a sort of Index, although an Index of a peculiar kind.... (CP 2. 249; emphasis added)

As mentioned before, the semiotic constitution, or structure, of the symbol, unlike that of the index, necessarily involves the "participation" of the interpretant-it is genuinely triadic: in practical terms this means that we have to know in advance what the object of the sign is in order to interpret it. When this is not the case, we have to learn it, whereas we are normally able to recognize the elements in a photograph or a painting. However, applying the implication principle by which the more complex subclasses involve the less complex, Peirce is explaining in the definition above that a symbol contains a sort of index, albeit of a "peculiar kind". We already know that an index incorporates an icon, which means that by transitivity a symbol contains some form of iconic material (were this not so, remember, we should be unable to perceive it - the symbol itself is perfectly general, while an index is instantaneous, and only the iconic traces in the sign are perceivable).

How, then, might an icon attain to symbolhood? Religious art abounds with examples, as evidenced by the numerous studies explaining the "hidden", i.e. symbolic, meanings of objects to be found in religious imagery: Hall (1983) is a good example. We learn, for example, that peacocks, which are to be found in much early religious imagery were placed there not for their esthetic value, but because their flesh was held by legend not to be subject to decomposition or putrefaction. In this way peacocks were not simply pictorially flamboyant birds but the symbols of everlasting life. Consider, as a further example, Van Eyck's Arnolfini Portrait, 1434, hanging in the National Gallery, London, which illustrates the principle perfectly. As a painting, the whole image is a sinsign by division one and an icon by the present one: it is an existent object composed of iconic material which we interpret to be a man and a woman holding hands, a dog carefully placed between them at their feet, a bed, a mirror, a pair of discarded clogs and a chandelier, etc. Although the meaning of the painting is disputed, it is thought to represent the wedding ceremony uniting the banker Giovanni Arnolfini and his wife. While it is impossible to establish definitively just what each carefully chosen object in the image actually represents, the dog is an interesting case of symbolism in the Peircean sense. At one level, it is simply a dog, possibly one in the house already or even a gift from husband to wife. However, it has also been interpreted as symbolizing fidelity between the two, an important feature of Christian marital doctrine.

The important point is that although an icon by definition, the painting contains pictorial symbols. They are instantiated in the objects the artist has elected to include: these are the indices of a "peculiar kind". What makes them symbols is the fact that this individual dog, for example, has been interpreted to represent something considerably more complex, namely a concept. All concepts are legisigns; they are general and have to be learned, and if it weren't for the various interpretations of the painting by art historians our understanding of the semiotic status of the dog would be all the poorer. Furthermore, the fact that interpretations of the painting and the meaning of the elements it contains are the object of disputes among art historians is evidence, too, of the symbolic status of the elements of the world behind the glass: they are almost universally deemed to mean more than their face value. 
However, there are less conventional cases of the symbolization of basically iconic material. Consider what might be termed "dysfunctional symbolization", the attribution to a painting, work of art or religious artifact of values not necessarily to be found in the elements in the representation itself; this is the case with most forms of iconoclasm, the destruction of paintings and sculptures by psyches disturbed by folly or fanaticism. There are numerous examples of art works which have suffered from attacks by knife or hammer: Ad Reinhardt's giant black paintings, Leonardo's Mona Lisa, and Michelangelo's Pietà are but three well known examples. Yet another form of iconoclastic re-symbolization occurs in the way certain artists appropriate paintings, etc. from earlier traditions in order to re-work them by investing them with new values, satirical or otherwise, e.g. Duchamp's treatment of the Mona Lisa, and Plate 5, below.

\section{Ecosemiotics}

With this section we enter the heart of the Peircean contribution to the semiotics of pictorial documents. It is at this point that the relation between his three categories and the principles of semiotics they govern is evidenced most spectacularly as the basis of an ecology of signs: no sign occurs in a vacuum, and as we saw above, in the practical process of human communication all signs have to be conveyed through, and by means of, an existential medium. This, after all, is the justification for Peirce having posited the category of replicas of legisigns (a Nominalist wouldn't have bothered). The replicas of speech, to take a simple example, carry through the air we breathe as a wave-like succession of peaks and troughs, while those we write, as Lessing, for one, was careful to emphasize, are a linear sequence of inscriptions on a page (but now also on the computer screen or some other equally visible medium). Were this medium not existential in nature, we should be unable to hear, see, feel or smell signs, nor should we be able to expunge them from a writing pad or a blackboard, for example. Most importantly of all, communication would be the prerogative of a happy band of telepaths.

It therefore seems not unreasonable to expect that the medium in which the sign is conveyed should have some bearing upon the form it presents, and that an ecology of signs, that is, a study of the relation between the sign, the medium through which it is transmitted, and the complexity of the object represented, should yield interesting information concerning the nature and determinations of that structure. It is within the context of this often neglected relation between sign and medium that we take up and extend the theme of the mode of representation of their objects by signs begun in the previous section. The main thrust of this section is, therefore, a preoccupation with a sign's iconicity, that is, its semiotic form, not simply because the study of the formal characteristics of signs is, as we shall see, determined in crucial ways by the relation between sign and medium, but also because such mediuminduced variations in form can be shown, for example, to be the major invariant element within the long history of discussions of metaphor and allegory in both image and text.

\section{The hypoicons}

We recall, first, that the object of a sign of human origin is nearly always going to be general (thoughts, volition, etc.), while the medium will be air (in the case of speech) or paper or a blackboard (if the sign is written), for example. These latter are cases of singularity, in other 
words, Peirce's Secondness. Thus in the case of Peirce's definition of semiosis, the object and interpretant are, in practice, more complex than the medium through which the sign is transmitted (as shown by the horizontal red line on Figure 1). His theory of the categories makes it possible to analyze these complex relations between medium, sign and object, the defining text of which is celebrated in paragraphs 2.276-7 of the Collected Papers in which he introduces the concept of the hypoicon.

2.276. ...But a sign may be iconic, that is, may represent its object mainly by its similarity, no matter what its mode of being. If a substantive be wanted, an iconic representamen may be termed a hypoicon. Any material image, as a painting, is largely conventional in its mode of representation; but in itself, without legend or label it may be called a hypoicon.

This relatively simple statement means that Plate 1 is, like any painting without a caption, a hypoicon, which, of course, doesn't preclude such pictorial signs from representing the complexity of legisigns. However, by applying the familiar three-way categorial analysis to the subclass of icons, Peirce distinguishes three distinct cases in the following terse definition: 2.277. Hypoicons may be roughly divided according to the mode of Firstness of which they partake. Those which partake of simple qualities... are images; those which represent the relations, mainly dyadic, or so regarded, of the parts of one thing by analogous relations in their own parts, are diagrams; those which represent the representative character of a representamen by representing a parallelism in something else, are metaphors.

Thus the categories of Firstness, Secondness and Thirdness determine three hypoiconic "situations":

- The case where the object is simply qualitative and therefore less complex than the medium through which the sign is transmitted.

- The case where the complexity of both object and sign corresponds to that of the medium.

- The case where the object represented by the sign is more complex than the medium (the object is general, the sign and medium are existential singularities).

In the first case, then, we say that the sign is an image, in the second a diagram, and in the third that the sign is a case of metaphor. We can illustrate these fundamental distinctions captured by Peirce's definition as they apply to a single theme, namely representations of the human face, by Plates 3, 4 and 5. In the two more complex cases, each illustration is accompanied by an explanatory diagram based upon Figure 1. The first is a black and white, cropped version of the cover illustration of volume two of Aubrey Beardsley's Yellow Book (1894); the second is a diagram of the human skull taken from Gray's Anatomy, while the third is a poster designed to stimulate church attendance in Britain at the end of the last century. Note that all the objects represented are to be found behind the semiotic window. 


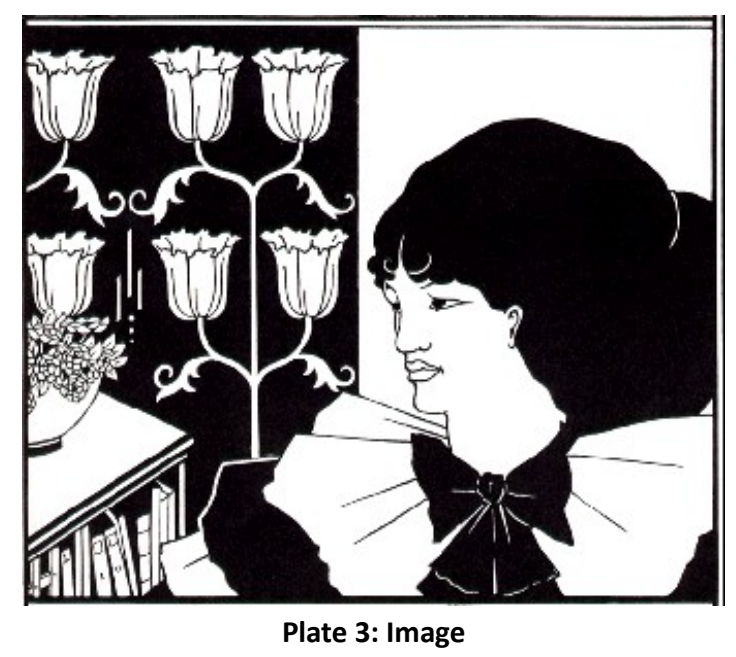

Plate 3 is a woodcut featuring among other things a stylized representation of a young woman's face, and Beardsley's principal concern is to organize these features in accordance with his particular esthetic preoccupations, e.g. the large expanses of black and yellow, the deliberate flattening of space and the rejection of perspective, as well as the deliberate placing of esthetic values above the representation of real live human features, etc. The actual relation between the eyes, nose and mouth are in this way of no scientific importance, and physiological accuracy was obviously not the artist's prime concern.

This is clearly not the case with Plate 4, which obviously targets surgeons, doctors and medical students: its primary purposes are to inform and to instruct, and physiological accuracy in this case is paramount, with the relations between the various parts of the skull scaled exactly in proportion and meticulously identified and labeled. Although it contains verbal legisigns of various kinds identifying the different parts of the skull (frontal, supraorbital foramen, etc., legisigns which obviously present no problems of comprehension to the experienced specialist), the mode of representation involved is inescapably iconic: in addition to these recognizable language signs the diagram is composed of lines forming relations within it which are intended to correspond term for term with relations among the parts of a human skull. In other words, as the definition states, for the sign to function correctly the dyadic or compound dyadic relations represented between the components in the diagrammatic representation have to correspond point by point, so to speak, to the relations between the components of the object represented. We don't know for certain that they do, but the sign represents itself to be representing them faithfully as doing so. This second representation, then, is an example of Peirce's diagram, an "icon of relations" (CP 4.418). 


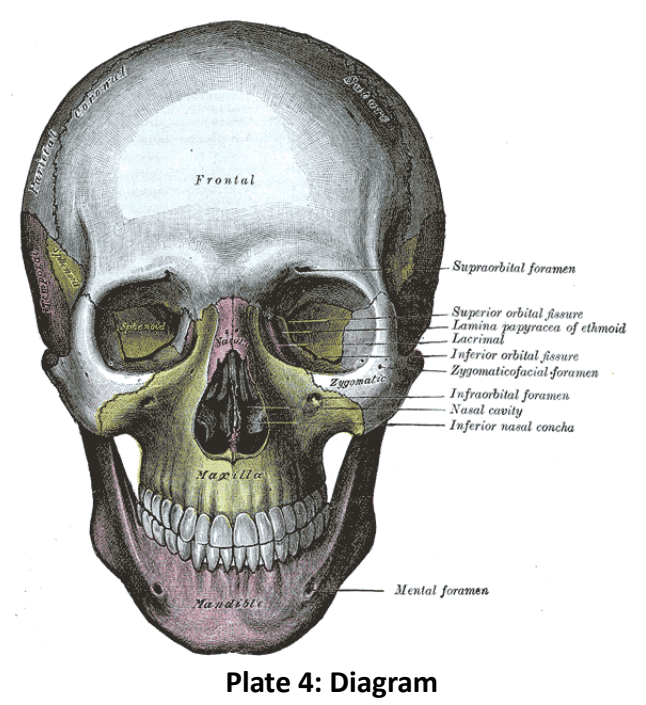

The accompanying diagram, Figure 2, shows the community of dyadic structure of both object and sign, and the transmission of such a sign through the existential Secondness of the medium is such that the sign can represent the structure of the object unhindered. This has been considerably simplified on the explanatory diagram, where the multiple dyadic relations between the parts of the skull and their representation have been reduced to two on Plate 4: for example, the spatial relation holding between the nasal cavity and the inferior nasal concha has been reduced to the single dyad $\mathbf{a}-\mathbf{b}$ in the object represented, determining the analogous dyad $\mathbf{a}$ '- $\mathbf{b}$ ' in its spatial representation in the sign. Note that, as in the case of the index, the structure of a diagrammatic sign is determined by the structure of the object and that the interpretant is not involved in that structural determination (though the interpretant is obviously involved in the semiosis).

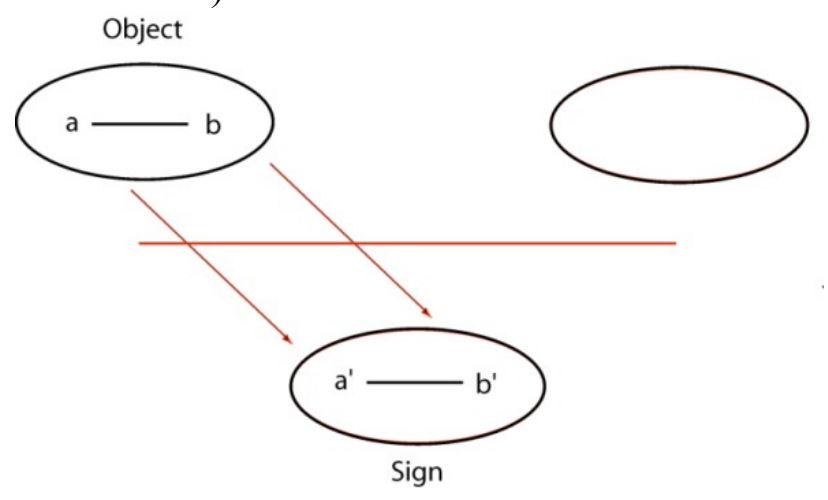

Figure 2: The semiotic constitution of the diagram

The purpose of the chimera on Plate 5, on the other hand, is of a different order, and there is clearly no scientific accuracy intended. This (originally multimodal) document entitled "(Meek Mild) Jesus: no wimp in a nightie" is offered as an example of pictorial metaphor. It was published in poster form as part of a campaign financed by the Church Advertising Network in Britain in preparation for Easter 1999, in an attempt to revamp the image of Christ and to revitalize church attendance at the time. It is, of course, yet another appropriation of the famous photograph of Che Guevara taken in 1960 by Alberto Korda, the idea being to replace the traditional docile image of a man prepared to turn the other cheek to his enemies and tormentors (whence the term "wimp", the "nightie" in question being an uncomplimentary reference to the ample robe Jesus is always represented as wearing) by a more aggressive and revolutionary one. 


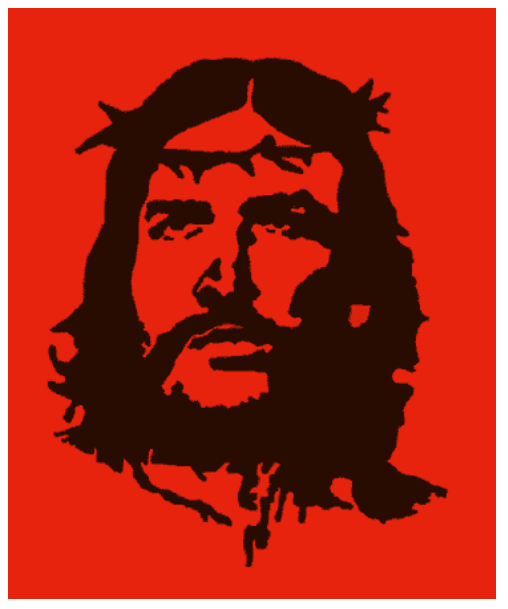

Plate 5: Metaphor

The interest of the poster is the way the features of two distinct but parallel ideologies, Marxist and Christian, are metaphorically integrated in a single image. What makes such an incongruous parallelism possible is the fact that both men were considered martyrs, and died for causes involving oppressors from home and abroad (Bolivians and American Army Special Forces on the one hand, and Jews and Romans on the other). The image of Christ is being targeted using the knowledge we are assumed to have of Che Guevara and the photograph as a basis for the comparison. The red background is common to both menblood and revolution. Thus, just three nonverbal features of expression and appearancereplicas of NVC legisigns - and the framing convention governing the slightly low-angled shot are sufficient for an efficient interpretation of the message.

The diagram on Figure 3 displays the parallelism in the object that the sign has to transmit through the existential medium, and its reconstruction in the interpetant if it is to be successfully interpreted by the congregation targeted. Within the parallelism the relation $\mathbf{a}-\mathbf{b}$ - c belongs to the unproblematic "base" domain (the terminology is not Peirce's, but that of Lakoff and Johnson 1981) assumed to be known to all, and representing, respectively, the beret, the facial expression and the low-angled shot from the Che Guevara photograph. The structure $\mathbf{a}$ '- $\mathbf{b}$ ' $-\mathbf{c}$ ', on the other hand, is the problematic domain targeted by the metaphor, here the revitalizing of the image of Christ. Within the parallelism, a and $\mathbf{a}$ ', $\mathbf{b}$ and $\mathbf{b}$ ', $\mathbf{c}$ and $\mathbf{c}$ ' are counterparts, that is, they correspond to each other within their respective domains: a and a' are respectively the beret and the crown of thorns, $\mathbf{b}$ and $\mathbf{b}$ ', the faces and expressions of Che Guevara and Christ, respectively, while c and ' ' can be supposed to be the framing techniques adopted by, respectively, the photographer and conventional artistic representations of Christ. The simplified structure of the resultant sign represents a reduction of the original two-tiered parallel to a "line" associating Christ's crown of thorns (a'), Che Guevara's face and moustache (b) and the particularity of the photographer's choice of frame (c). 


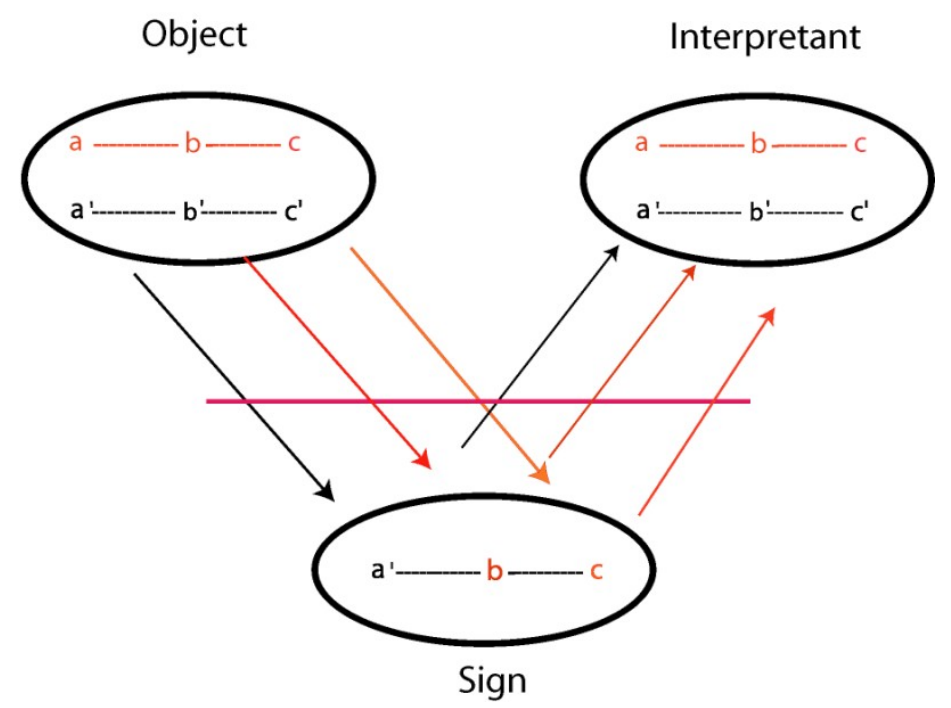

Figure 3: The semiotic constitution of metaphor

It is thus characteristic of metaphorical signs, firstly, that they should be underspecified with respect to the parallelism they have to convey-the existential structure of the medium prevents the transmission of the two-tiered totality of the original objet, and this parallelism has to be inferred at the interpretation stage; and secondly, that such signs always, at least when first encountered, appear incongruous - the incomplete blending of elements from two distinct domains of experience produces the sort of chimera shown on Plate 5. This is no less true of linguistic metaphor than of the nonverbal type discussed here. In this way, Peirce's category theory offers a neat explanation of how the medium crucially determines the structure of signs of this type, whether verbal or pictorial.

Finally, the diagram below illustrates in linear fashion this constricting "funnel" effect of the Secondness of the medium on the complexity of the object being represented: the parallelism in the object has been reduced unavoidably to a single "line" in the sign, with a consequent loss of information, and an incongruity within the sign itself.

Object

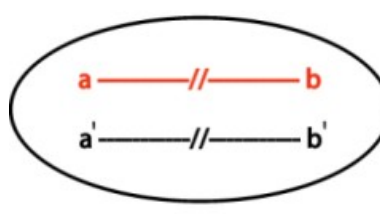

Sign

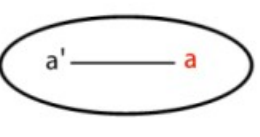

Interpretant

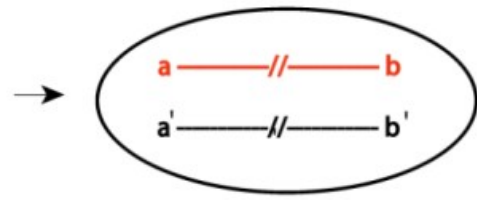

Figure 4: The funnel effect of Secondness on the structure of the sign

Within the object, the vector $\mathbf{a}-\mathbf{b}$ represents elements from the base domain, the unproblematic area of experience assumed to be common to both speaker and listener; the vector a'-b', on the other hand, represents the problematic domain being "assessed" in the target domain. Thus in the sentence Achilles is a lion, the lion, renowned as the king of the jungle, i.e. adjudged by legend to be the most fearsome beast in the animal world, is the base, while Achilles and his value in relation to other human warriors is the target. The sign in this case contains the relation $\mathbf{a}$ - $\mathbf{a}$, that is, the element Achilles from the target domain, here represented for simplicity as a', plus lion, here a, from the base, while the other elements of the parallelism, namely the other animals (b) and the other human warriors (b') have perforce been omitted from the sign. 


\section{Beyond metaphor}

Finally, the hypoiconic structure of allegory and personification, here an illustration from an emblem book, is more complex than the case of metaphor examined above, although this is not immediately apparent. Emblems were typically composed of a motto, an image or pictura, and a text in verse and/or an epigram. In this case, the pictura has been extracted from Andrea Alciato's 16th century Book of Emblems, and represents the emblem Vigilantia et custodia. In appearance it seems to be far simpler than the skull from Gray's Anatomy, being composed of easily recognizable facing cockerels perched on bronze bells at the top of twin towers, one in the dark, the other in the light, together with two lions guarding the entrance to a temple. We are tempted to think that even a young child could successfully interpret such an image.

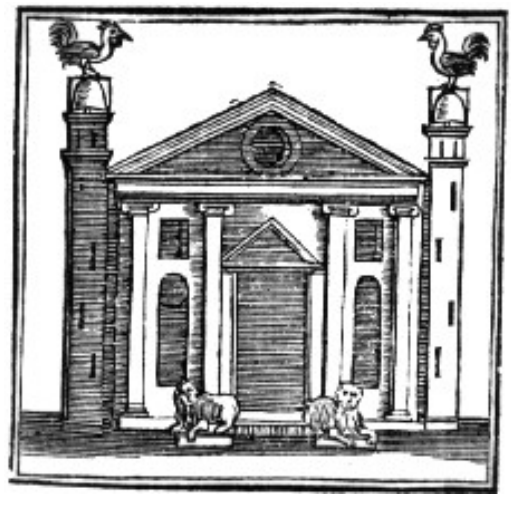

Wakefulness and watchfulness

The crowing cock - because it gives signs of the coming Dawn and recalls toiling hands to a new day's labor; the bronze bell - because it calls the wakeful mind to higher things: each is fashioned on sacred towers. And here's a lion - but because this guardian sleeps with open eyes, it's therefore placed before the temple doors.

Plate 10: Alciato's emblem 16

However, as the experienced adult knows, following a long tradition of fables and bestiaries from Æsop and Jean de La Fontaine to James Thurber and Art Spiegelman, the animals are intended to represent human qualities, here wakefulness and watchfulness (and if he doesn't, the epigram is there to help). In other words, this picture represents an object far more complex than itself, a parallelism, in fact drawn between two pairs of animals and two commendable human qualities. The structure of the picture then is that of Peirce's metaphor, but the contents of the sign are even more underspecified than in the canonical case of metaphor. This situation we can diagram using the funnel schema from above:

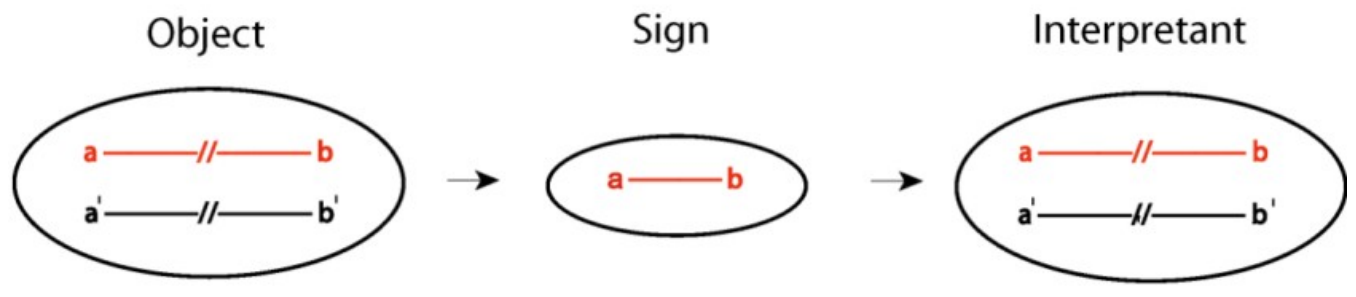

Figure 5: The hypoiconic structure of allegory

If we compare this schema with Figure 4 we see that the pictura itself contains only elements from the base domain, namely pairs of lions and cockerels. The two human qualities being targeted have to be inferred from our collateral experience of the genre. This (much simplified) would be the case, too, in the graphic novel Maus, where the vector $\mathbf{a}-\mathbf{b}$ would stand for the well-known relation holding between cats and mice, while a'-b' would represent their respective counterparts in the target, namely the Nazis and the Polish Jews. 
However, within the sign on Figure 5, as in the novel, the base relation between cats and mice is the only one to be fully represented, leaving the target to be inferred by the reader. In this way, fabulists and allegorist the world over, have, through the ages, appropriated and reworked the structure of metaphor as Peirce identifies it in paragraph 2.277.

\section{Image and information}

\section{Information}

The final trichotomy of the 1903 classification discriminates between the three ways in which signs afford information about their objects, and is no less important to a visual semiotics than the others. Now Peirce states that the "only way of directly communicating an idea is by means of an icon" (CP 2.278), which raises the interesting question as to how icons, in particular pictorial icons, actually do this since, as seen above, they are composed principally of qualities. We note, to begin with, that outside computer science with its bits and bytes the term "information" is usually understood to mean some descriptive proposition, assertion or statement enabling us to describe some system. For example, a proposition such as Collioure has an interesting church, composed of the subject Collioure and the predicate has an interesting church, enables us to describe if only in part the "system" Collioure. Note that, in addition to the traditional formula of Subject + Predicate, Peirce defines the proposition as being composed of an index, which indicates what the proposition is about and establishes a universe of existence or ontology, and an icon from which information about the index can be obtained. The problem for a visual semiotics at this point, then, is a) to determine just what sort of information can be obtained from images of all sorts, and b) to what extent images can function propositionally.

\section{The hypoicons once more}

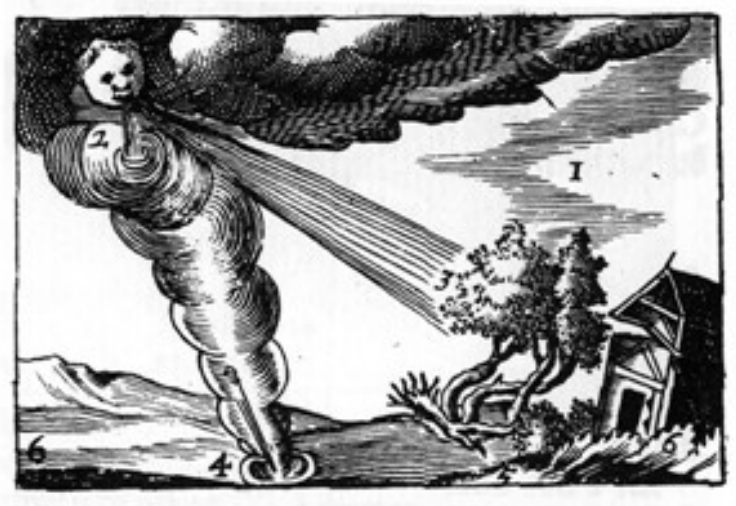

Plate 6: "Air" from the Orbis pictus

One way of obtaining information from images is illustrated by the fundamental differences between Plates 6 and 7. The first is an illustration from Comenius's Orbis sensualium pictus, an innovative 17 th century pedagogical aid composed of chapters of images and associated "nomenclatures", and constructed on the Aristotelian principle, explicitly stated in the work's Introduction, that there can be nothing in the "understanding" that isn't first in the senses, the idea being that the visible world is either there before the child's eyes, or recognizable from past experience in the form of a mental image already in his mind, and that associating the 
known image-content with the as yet unlearned word can lead to the extension of a child's vocabulary. Each chapter is composed of a woodcut illustrating one of the one hundred and fifty themes, with, on the facing page a list of terms in Latin. The second, Plate 7, is a cutaway diagram of a diesel pump. Both display an arrangement of distinct parts, each of which is identified with a number.

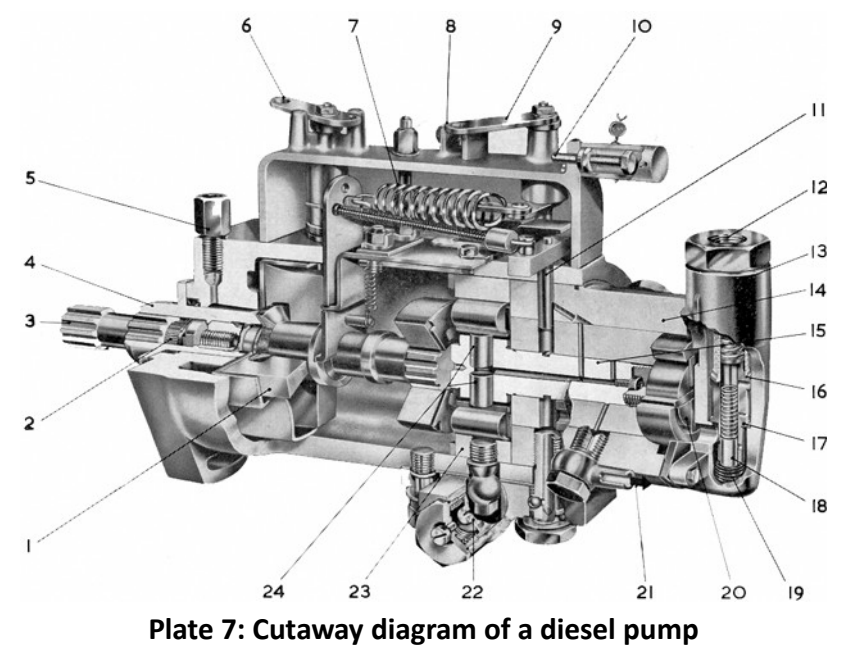

However, there the similarity ends, for while it would theoretically be possible to rearrange certain items on Plate 6, e.g. by placing the trees behind the house, without destroying its purpose, such a rearrangement would be totally impossible in the case of the pump. The explanation is simple: while both illustrations are iconic, Plate 6 is an image in the technical Peircean sense, composed simply of relatively unordered qualities (the wind must be directed at the trees, of course), Plate 7 is structured as a diagram, in the Peircean sense here too, in that relations between parts in the object, namely the pump, strictly determine analogous relations visible in the sign. This is, of course, the principle behind geometrical diagrams and the wordless instructions on how to install electrical and other appliances in the home; it is also, of course, the principle illustrated by the diagram from Gray's Anatomy. While such signs don't actually tell us anything as a proposition would, they nevertheless afford valuable, viable information.

\section{Propositional imagery}

However, there is more to the problem than the essential differences holding between imagic and diagrammatic icons. Indeed, more important for present considerations is the fact that, for Peirce, the whole notion of information was a function of a sign's capacity to indicate the existence of its object. For example, given the phenomenological status of the index as described earlier, in a proposition such as the one given above, Collioure, a proper noun, is the readily identifiable subject of the proposition: it is represented to the interpretant to be the index of some object existing independently of the sign within a specific ontology. This may or not be so, but the utterance represents this to be the case, and for this reason such a sign, with its "double" syntax (i.e. composed of two elements, namely a subject and a predicate) is subject to the principle of contradiction: it is either true or false. In such cases, Peirce identifies the sign as "dicent", i.e. a sign that actually tells us something.

Moving to pictorial data, a captionless photograph of the entity Collioure is "dicent" to the extent that, as an index, it displays and thus guarantees the existence at some unspecified 
moment of such an object (or purports to, even in the case of digitally modified images). This cannot be the case with an icon, which is composed simply of qualities (aided in the case of diagrams by indices of a special sort), and so an icon, the object of which is necessarily whatever happens to resemble it — an "imaginary object" is one of Peirce's terms - can only denote the possibility, not the existence, of its object and cannot ipso facto convey information. In other words, they have an informative potential but, as a consequence of their qualitative status, they cannot tell us anything. With this in mind we turn to illustrations of the sign-types defined by this third trichotomy within which, in order of increasing complexity, Peirce distinguishes between rhemes, dicent signs and arguments, which differ essentially in the peculiar syntax connecting them to the object represented.

\section{Rhemes}

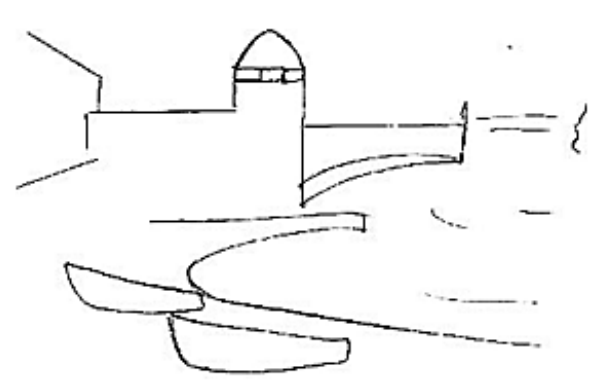

Plate 8: A Catalan fishing port

Of the several examples from this section, Plate 8, a sketch of a fishing village beloved of Matisse and the Fauves has the lowest informational value. Composed simply of lines and shapes, it is both a singular sign and an icon. Since there is no physical connection between the port and its pictorial representation, as there would be if it had been a photograph, it is in no way indexical, it has a simple syntax (only the sign is involved), it cannot form a proposition and therefore does not enable us to describe any system. Peirce called such signs rhemes - simple, substitutive signs. The sketch is a rheme, as is the expression above - has an interesting church. Nevertheless, although they do not constitute propositions, information of the sort mentioned in the discussion of Plates 6 and 7 can be obtained from such signs.

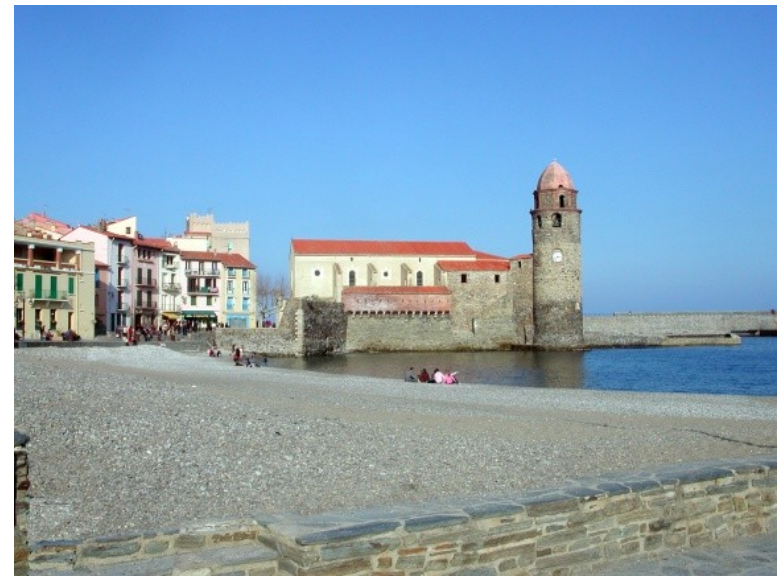

Plate 9: The church at Collioure

Plate 9, the photograph of the church in the same Catalan fishing village, is necessarily an index, since it stands in an existential relation to this part of the town (someone took the photograph at a particular time). It is also visibly iconic since we can, from the lines, forms 
and colors composing it, recognize it as something like a church or a lighthouse, even if we've never seen the entity in question (it is also iconic by virtue of the implication principle associating all indices with some form of incorporated icon).

Nevertheless, the syntax of this particular sign is no less double than a proposition associating a subject and a predicate such as Collioure has an interesting church: the index is the fact that a photographer took the photograph at a particular date, and the whole image "tells" us that "there exists (or existed) something like this", the "like this" being, of course, the iconic material visible in the plate. In other words, such a sign, while still dicent, is less informative in that the index is not as clearly identifiable as the subject is in a linguistic proposition: we simply understand that the physical connection between the port and the film did in fact take place at some unspecified and unspecifiable time, but the port itself is not identified by name.

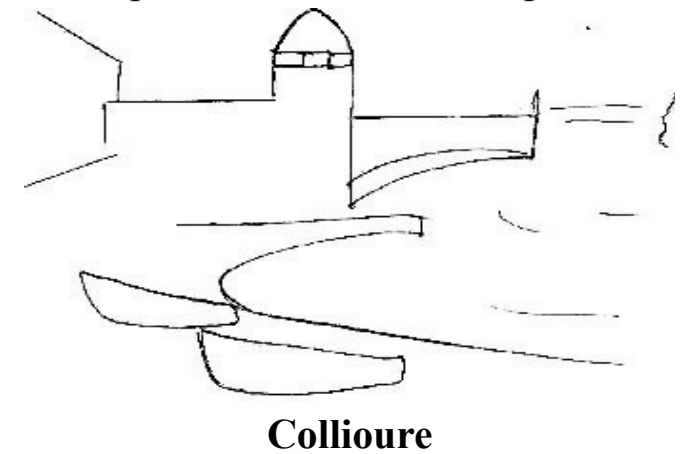

Plate 10: A sketch of the port of Collioure plus caption

However, Plate 10, which is a sketch of the said church plus a caption beneath, is, unlike Plate 8 , a double sign - a sort of proposition, therefore, by virtue of its characteristic "double" syntax. The caption is composed of a proper noun (Collioure), which is a type of index, plus an icon formed by the sketch (identical to the one on Plate 8). Thus the association of the index and the icon "informs" us that the existent entity Collioure is somehow like the lines, etc., on the sketch. Moreover, although composed of the simple qualities of a line drawing, as a consequence of the presence of the legisign Collioure, Plate 10 is semiotically more complex than the photograph on Plate 9.

Finally, returning briefly to some of the previous examples, we see that the NVC legisigns on Plates 1 and 2 are necessarily rhematic: being iconic and having therefore a "simple" syntax, they lack the dyadic, existential requirement that would enable them to yield viable information. Similarly, the Van Eyck dog and the peacock are both rhematic symbols for the same reason: their syntax, too, is simple.

\section{Argument}

Finally, the argument, the "triple, or rationally persuasive sign", the most complex of all subclasses, and the means of discovery of new information, is customarily linguistic in nature, as only language signs readily admit of the sort of complex articulation generally required by overt ratiocination. However, owing perhaps to changes of attitude towards the notion of mental images and to advances in digital technology, Peirce's enthusiasm for diagramming the reasoning process - he wrote of his Existential Graphs that they were "My Chef d'œuvre"seems to have been vindicated by a recent renewal of interest in both diagrammatic and heterogeneous, or multi-modal logics, that is, logics which combine visual information and 
symbolization. However, to assess the Graphs in relation to the present problematic would require a whole book, not an article, and the interested reader can find a very full account of this aspect of Peirce's preoccupation with the iconic, and a sympathetic, easily readable introduction to Peirce's three systems of graphs in Shin (2002) and its references.

\section{Conclusion}

What may have surprised the reader of the foregoing lines is the absence of any sort of recipe concerning the purpose of pictorial signs: the semiotic system described above is a logic, an empty formalism, while the purpose to which a sign may be put is the province of rhetoric. This is because for Peirce this particular semiotics and rhetoric were the two polar branches of his philosophy of representation: the first he described as a "speculative grammar", that is, a theoretical syntax whose purpose is not to stipulate what a given sign does or does not mean, but rather the conditions a) in which some entity can function as a sign, b) how any such sign is able to signify, and c) to what extent it can afford information. As a scientist formed in the laboratory he was keenly aware of the need for scientific accuracy in the acquisition and subsequent representation of knowledge. His speculative grammar was the first step in this undertaking, and the present article is an attempt to show how the discipline required of such a task might apply to pictorial data. "Speculative rhetoric", on the other hand, the final, most specialized branch of the philosophy of representation, "is the doctrine of the general conditions of the reference of symbols and other signs to the interpretants which they aim to determine", (CP 2.93). In other words, within a Peircean perspective, semiotics and rhetoric are distinct branches of a veritable philosophy in which semiotics provides the doctrine of rhetoric with its theoretical "syntactical" prerequisites. Although Peirce has provided semioticians with considerable material concerning the semiotics, there is far less available for his theory for speculative rhetoric. It is to be hoped that the foregoing will stimulate interest in this largely uncharted research undertaking. 


\section{References}

Argyle, M., (1972), "Non-verbal communication", in Non-Verbal Communication, Hinde, R., A, (ed.), London: Cambridge University Press, pp. 243-269.

Argyle, M., (1988), Bodily Communication, 2nd edition, London: Methuen.

Guiraud, P., (1980), Le Langage du corps, Paris: Presses Universitaires de France

Hall, J., (1983), Hall's History of Ideas and Images in Italian Art, London: John Murray.

Jappy, T., (2010), Regards sur le poème muet: petite introduction à la sémiotique visuelle peircienne, Perpignan : Presses universitaires de Perpignan.

Kendon, A., (1980), (ed.), Nonverbal Communication, Interaction and Gesture, The Hague: Mouton.

Lakoff, G. and M. Johnson, (1980), Metaphors We Live By, Chicago: University of Chicago Press.

Lessing, G., (1766 [1984]), Laocoön: An Essay on the Limits of Painting and Poetry, Baltimore: Johns Hopkins University Press

Peirce, C. S., (1931-58), Collected Papers, 4 Vols., Hartshorne, C., P. Weiss and A. Burks, (eds), Cambridge, Mass.: Harvard University Press.

Peirce, C. S., (1998), The Essential Peirce - Volume 2: Selected Philosophical Writing: (18931913), Peirce Edition Project, Bloomington: Indiana University Press.

Schapiro, M., (1994), Theory and Philosophy of Art: Style, Artist, and Society, New York: Brazillier.

Shin, S.-J., (2002), The Iconic Logic of Peirce's Graphs, Cambridge, Mass.: MIT Press. 